\title{
Qualitative aspects in dual-phase-lag heat conduction
}

\author{
RAMÓN QUINTANILLA ${ }^{1}$ \\ Department of Applied Mathematics II \\ UPC Terrassa, Colom 11, 08222 Terrassa, Spain \\ ramon.quintanilla@upc.edu \\ REINHARD RACKE ${ }^{2}$ \\ Department of Mathematics and Statistics \\ University of Konstanz \\ 78457 Konstanz, Germany \\ reinhard.racke@uni-konstanz.de
}

Abstract: We consider the system of dual-phase-lag heat conduction proposed by Tzou [21]. First, we prove that the solutions of the problem are generated by a semigroup of quasicontractions. Thus, the problem of third-order in time is well-posed. Then the exponential stability is investigated. Finally the spatial behavior of solutions is analyzed in a semi-infinite cylinder.

\section{Introduction}

It is well known that the usual theory of heat conduction based on Fourier's law predicts infinite heat propagation speed. Heat transmission at low temperature has been observed to propagate by means of waves. These aspects have caused intense activity in the field of heat propagation. Extensive reviews on the so-called second sound theories (hyperbolic heat conduction) are given in Chandrasekharaiah [2] and in the books of Müller and Ruggeri [14] and Jou et al. [13]. A theory of heat conduction in which the evolution equation contains a third order derivative with respect to time was proposed in [5]. Several instability results have been obtained for the theory, see e.g. $[4,15]$, as well as proof of the nonexistence of global solutions in the nonlinear theory [19]. In 1995, Tzou [21] proposed a theory of heat conduction in which the Fourier law is replaced by an approximation of

\footnotetext{
${ }^{0}$ AMS subject classification: 35 L 35, 74 F 05, 74 G 50

Keywords and phrases: parabolic model in heat conduction, well-posedness, spatial evolution in a semi-infinite cylinder, exponential stability

${ }^{1}$ Supported by the project "Aspects of stability in thermomechanics" (BFM/FEDER 2003-00309)

${ }^{2}$ Supported in the DFG-project "Hyperbolic Thermoelasticity" (RA 504/3-1).
} 
the equation

$$
\mathbf{q}\left(\mathbf{x}, t+\tau_{q}\right)=-k \nabla \theta\left(\mathbf{x}, t+\tau_{\theta}\right), \quad \tau_{q}>0, \tau_{\theta}>0,
$$

where $\tau_{q}$ is the phase-lag of the heat flux and $\tau_{\theta}$ is the phase-lag of the gradient of temperature. The relation (1.1) states that the gradient of temperature at a point in the material at time $t+\tau_{\theta}$ corresponds to the heat flux vector at the same point at time $t+\tau_{q}$. The delay time $\tau_{\theta}$ is caused by microstructural interactions such as phonon scattering or phonon-electron interactions. The delay $\tau_{q}$ is interpreted as the relaxation time due to fast-transient effects of thermal inertia.

Instead of Fourier's law, being equivalent to assuming

$$
\tau_{q}=\tau_{\theta}=0
$$

and leading to the paradoxon of infinite propagation speed, we consider the model proposed by Tzou [21], where

$$
\tau_{q}>0, \quad \tau_{\theta}>0
$$

are positive relaxation times, and where a second-order approximation for $\mathbf{q}$ and $\theta$ is used, turning (1.1) into

$$
q_{i}+\tau_{q} \dot{q}_{i}+\frac{\tau_{q}^{2}}{2} \ddot{q}_{i}=-k \theta_{, i}-k \tau_{\theta} \dot{\theta}_{, i}-\frac{\tau_{\theta}^{2}}{2} \ddot{\theta}_{, i}
$$

Thus, in this paper, we consider the theory developed by taking a Taylor series expansion on both sides of (1.1) and retaining terms up to the second-order in $\tau_{q}$ and $\tau_{\theta}$. This equation has not received much attention in the literature (until now). However, we can recall that the spectrum was studied in [18].

A natural question is the determination of the time parameters $\tau_{q}$ and $\tau_{\theta}$ (see [7]) and our work is motivated by this question. One might expect that mathematical analysis of existence, uniqueness and stability issues, for example, would furnish certain restrictions on the parameters. One property to hold for solutions of a heat equation should be exponential stability (or at least stability). In [18], stability (for the heat conduction) was established whenever

$$
\frac{\tau_{\theta}}{\tau_{q}}>\sqrt{2}-1
$$

Thus, under condition (1.4), one has a heat theory with a third-order derivative in time in the equation that predicts stability. This is of interest in the light of the results obtained in the theory proposed in [5]. By means of several exact solutions instability of solutions was also established in [18] whenever the condition (1.4) is violated. Thus, one may assume that the condition (1.4) must be satisfied in order to use this model to describe heat transmission.

In this paper we study four kinds of questions. One is to determine the suitable frame where the third-order problem of heat conduction is well posed. Second is to prove the 
exponential stability. Third to determine the spatial behavior of the solutions of the heat conduction in a semi-infinite cylinder in $\mathbb{R}^{3}$. The last goal is to obtain a uniqueness result in case of unbounded domains and when we assume that the solutions can be unbounded at infinity.

It is worth recalling that in case that we only consider the development until first order in $\tau_{\theta}$ we obtain an hyperbolic theory which has been studied and analyzed in $[12,16,17]$. Our aim here is to continue the clarification of these theories and to see what happens in the new theory. We believe that the mathematical analysis will help us to understand the applicability of these theories.

This paper is organized as follows: in Section 2 we settle the field equations and the boundary and initial conditions of the problem we consider in this paper. A uniqueness and existence result is proved in Section 3. In Section 4 we prove the exponential stability. In Section 5, we obtain some results of Saint-Venant's type concerning the spatial behavior of solutions in a semi-infinite cylinder and some improvements of them as obtained in Section 6. The last section is devoted to the uniqueness of solutions when the solutions can grow in a fast way at infinite.

We shall denote the three-dimensional semi-infinite cylinder by $R$, with cross-section $D$. The finite end face of the cylinder is in the plane $x_{3}=0$. The boundary $\partial D$ is supposed regular enough to allow the use of the divergence theorem. We denote by $R(z)$ the set of points of the cylinder $R$ such that $x_{3}$ is greater than $z$ and by $D(z)$ the cross-section of the points such that $x_{3}=z$.

\section{Preliminaries}

In this paper we study solutions $\theta=\theta(\mathbf{x}, t))$ of the heat equation

$$
k \hat{\theta}_{, i i}=\dot{\tilde{\theta}}
$$

We have used the notation

$$
\hat{f}=f+\tau_{\theta} \dot{f}+\frac{\tau_{\theta}^{2}}{2} \ddot{f}, \quad \tilde{f}=f+\tau_{q} \dot{f}+\frac{\tau_{q}^{2}}{2} \ddot{f},
$$

where $\tau_{\theta}>0, \tau_{q}>0$ are the dimensionless time lag parameters.

We study the qualitative behavior of classical solutions subject to the initial conditions

$$
\theta(\mathbf{x}, 0)=\theta^{0}(\mathbf{x}), \dot{\theta}(\mathbf{x}, 0)=\vartheta^{0}(\mathbf{x}), \ddot{\theta}(\mathbf{x}, 0)=\phi^{0}(\mathbf{x}),
$$

and the boundary conditions

$$
\begin{aligned}
\theta(\mathbf{x}, t) & =0, \mathbf{x} \in \partial D \times[0, \infty) \\
\theta\left(x_{1}, x_{2}, 0, t\right) & =g\left(x_{\alpha}, t\right) \text { on } D(0) \times[0, \infty),
\end{aligned}
$$


where the prescribed boundary data $g\left(x_{\alpha}, t\right)$ on the end $x_{3}=0$ is such that $g\left(x_{\alpha}, 0\right)=$ $\theta^{0}\left(x_{\alpha}\right)$ and $g$ are assumed to vanish on $\partial D(0) \times[0, \infty)$.

Observe that in the limit as $\tau_{\theta}$ and $\tau_{q}$ tend to zero,. we recover from (2.1) the usual heat equation. In this limit, the existence, stability and the spatial evolution of solutions has been studied in a variety of contexts. When $\tau_{q}$ and $\tau_{\theta}$ are positive the results to be described in the sequel will be seen to be similar to those obtained previously for such equations.

In some parts of the paper (see Section $4,6,7$ ), we will assume that $\tau_{\theta}>\tau_{q}$. We note that from the empiric point of view this is a rational assumption as it can be seen in $[21,22]$. We note that our equation can be written as

$$
\hat{\theta}_{, i i}=A \dot{\hat{\theta}}+B \ddot{\theta}+C \dot{\theta}
$$

where

$$
A=\frac{\tau_{q}^{2}}{\tau_{\theta}^{2}}, B=\tau_{q}-\frac{\tau_{q}^{2}}{\tau_{\theta}}, C=1-\frac{\tau_{q}^{2}}{\tau_{\theta}^{2}} .
$$

As $\tau_{\theta}>\tau_{q}$ the constants $B, C$ are positive.

In the course of our calculations, we will use the knowledge of the eigenvalues of the real symmetric positive definite matrix

$$
\left(\begin{array}{ccc}
a & 0 & b \\
0 & m & 0 \\
b & 0 & l
\end{array}\right)
$$

It is clear that one of the eigenvalues is $m$ and the others are the eigenvalues of the matrix

$$
\left(\begin{array}{ll}
a & b \\
b & l
\end{array}\right)
$$

The eigenvalues of this matrix are

$$
\lambda^{ \pm}=\frac{1}{2}\left(a+l \pm \sqrt{(a-l)^{2}+4 b^{2}}\right)
$$

so that the smallest eigenvalue is:

$$
\lambda^{-}=\frac{1}{2}\left(a+l-\sqrt{(a-l)^{2}+4 b^{2}}\right) .
$$

When

$$
\gamma>\frac{\left(\tau_{q}^{2}-\tau_{\theta}^{2}\right)^{2}}{2 \tau_{q}^{2} \tau_{\theta}^{2}\left(\tau_{\theta}+\tau_{q}\right)}
$$


the matrix

$$
J:=\left(\begin{array}{ccc}
\gamma^{-1}+\frac{\tau_{q}+\tau_{\theta}}{2} & 0 & \frac{\tau_{q}^{2}+\tau_{\theta}^{2}}{4 \gamma} \\
0 & \frac{\tau_{q} \tau_{\theta}\left(\tau_{\theta}+\tau_{q}\right)}{4}+\frac{\tau_{\theta} \tau_{q}}{\gamma} & 0 \\
\frac{\tau_{q}^{2}+\tau_{\theta}^{2}}{4 \gamma} & 0 & \frac{\tau_{\theta}^{2} \tau_{q}^{2}}{4 \gamma}
\end{array}\right)
$$

is defined positive and the minimum eigenvalue can be calculated by means of the arguments proposed previously. We shall denote by $\lambda_{\gamma}$ the smaller eigenvalue.

\section{Well-posedness}

We shall formulate the problem for the semi-infinite cylinder $R$ in three space dimensions, but the well-posedness holds for general domains.

The well-posedness result for this system - not being trivial for third-order in time systems, cp [6, p.125] — can be achieved by an appropriately sophisticated choice of variables and spaces which reflect the special structure of the system.

We first transform the system (2.1)-(2.5) to zero boundary conditions on all of $\partial R$ by defining

$$
\psi\left(x_{\alpha}, 0, t\right):=\theta\left(x_{\alpha}, 0, t\right)-g\left(x_{\alpha}, t\right), \quad \psi\left(x_{\alpha}, x_{3}, t\right):=\theta\left(x_{\alpha}, x_{3}, t\right) \quad \text { for } x_{3}>0,
$$

and using $\theta$ instead of $\psi$ again, we obtain the initial boundary value problem

$$
\begin{gathered}
k \hat{\theta}_{, i i}=\dot{\tilde{\theta}}-p, \\
\theta(\mathbf{x}, 0)=\theta^{0}(\mathbf{x}), \dot{\theta}(\mathbf{x}, 0)=\vartheta^{0}(\mathbf{x}), \ddot{\theta}(\mathbf{x}, 0)=\phi^{0}(\mathbf{x}), \\
\theta(\mathbf{x}, t)=0, \mathbf{x} \in \partial R \times[0, \infty)
\end{gathered}
$$

where the given heat supply $p$ arise from the transformation (3.1) in terms of the boundary data $g$.

We remark that finding a solution $\theta$ to (3.2)-(3.4) allows to determine the desired solutions $\theta$ of the original system.

Defining

$$
\mathbf{V}:=\left(\theta, \theta_{t}, \theta_{t t}\right)^{\prime}
$$

we obtain

$$
\mathbf{V}_{t}=A \mathbf{V}+\mathbf{F}, \quad V(0)=V^{0}
$$

with the (yet formal) differential operator $A$ given by the symbol

$$
A_{f}:=\left(\begin{array}{ccc}
0 & 1 & 0 \\
0 & 0 & 1 \\
\frac{2 k}{\tau_{q}^{2}} \Delta & \frac{2}{\tau_{q}^{2}}\left(k \tau_{\theta} \Delta-1\right) & \frac{k \tau_{\theta}^{2}}{\tau_{q}^{2}} \Delta-\frac{2}{\tau_{q}}
\end{array}\right)
$$


the right-hand side $\mathbf{F}$ given by

$$
\mathbf{F}:=(0,0, p)^{\prime}
$$

and the initial value

$$
\mathbf{V}^{0}(\mathbf{x}):=\left(\theta, \theta_{t}, \theta_{t t}\right)^{\prime}(\mathbf{x}, 0)
$$

with its components being given in terms of the originally prescribed initial data in (3.3) by using the differential equations.

As underlying Hilbert space we choose

$$
\mathcal{H}:=H_{0}^{1}(R) \times H_{0}^{1}(R) \times L^{2}(R)
$$

with inner product

$$
\begin{aligned}
\langle V, W,\rangle_{\mathcal{H}}:= & \frac{k}{4}\left(\tau_{q}+\tau_{\theta}\right) \tau_{q} \tau_{\theta}\left\langle\nabla V^{2}, \nabla W^{2}\right\rangle+\frac{k}{2}\left(\tau_{q}+\tau_{\theta}\right)\left\langle\nabla V^{1}, \nabla W^{1}\right\rangle \\
& +\frac{1}{2}\left\langle V^{1}+\tau_{q} V^{2}+\frac{\tau_{q}^{2}}{2} V^{3}, W^{1}+\tau_{q} W^{2}+\frac{\tau_{q}^{2}}{2} W^{3}\right\rangle
\end{aligned}
$$

where $\langle\cdot, \cdot\rangle$ denotes the usual $L^{2}(R)$-inner product. The operator $A$ is now given as

$$
A: D(A) \subset \mathcal{H} \mapsto \mathcal{H}, \quad A V:=A_{f} V
$$

with

$$
D(A):=\left\{V \in \mathcal{H} \mid A_{f} V \in \mathcal{H}\right\}=D(A):=\left\{V \in\left(H_{0}^{1}(R)\right)^{3} \mid \Delta V^{j} \in L^{2}(R), j=1,2,3\right\}
$$

Lemma 3.1 There exists a constant $c_{1}>0$ such that for all $V \in D(A)$

$$
\left|\langle A V, V\rangle_{\mathcal{H}}\right| \leq c_{1}\|V\|_{\mathcal{H}}^{2}
$$

holds.

Proof: We have (without loss of generality for a real inner product)

$2\langle A V, V\rangle_{\mathcal{H}}=-k\left(\frac{\tau_{q}^{2} \tau_{\theta}^{2}}{4}\left\langle\nabla V^{3}, \nabla V^{3}\right\rangle+\tau_{q} \tau_{\theta}\left\langle\nabla V^{2}, \nabla V^{2}\right\rangle+\left\langle\nabla V^{1}, \nabla V^{1}\right\rangle+\frac{1}{2}\left(\tau_{q}^{2}+\tau_{\theta}^{2}\right)\left\langle\nabla V^{3}, \nabla V^{1}\right\rangle\right)$

which was possible because of the specific choice of the inner product. Since

$$
\left\langle\nabla V^{3}, \nabla V^{1}\right\rangle \leq \frac{\epsilon}{2}\left\langle\nabla V^{3}, \nabla V^{3}\right\rangle+\frac{1}{2 \epsilon}\left\langle\nabla V^{1}, \nabla V^{1}\right\rangle,
$$

where $\epsilon$ is an arbitrary positive constant, and if we take

$$
\epsilon=\frac{\tau_{q}^{2} \tau_{\theta}^{2}}{\tau_{q}^{2}+\tau_{\theta}^{2}}
$$


we see that

$$
2\langle A V, V\rangle_{\mathcal{H}} \leq k\left(\frac{\left(\tau_{q}^{2}+\tau_{\theta}^{2}\right)^{2}}{4 \tau_{q}^{2} \tau_{\theta}^{2}}-1\right)\left\langle\nabla V^{1}, \nabla V^{1}\right\rangle
$$

which implies the assertion with

$$
c_{1}:=\frac{\left(\tau_{q}+\tau_{\theta}\right)\left(\tau_{q}-\tau_{\theta}\right)^{2}}{4 \tau_{q}^{2} \tau_{\theta}^{2}} .
$$

QED

As a consequence we see that for $d>c_{1}$ the operator $A-d$ is dissipative and invertible.

Lemma 3.2 For all $d>c_{1}$ we have that the range of $A-d$ is all of $\mathcal{H}$.

Proof: The solvability of $(A-d) V=F$ is equivalent to solving

$$
\begin{aligned}
V^{2}-d V^{1} & =F^{1}, \\
V^{3}-d V^{2} & =F^{2}, \\
\frac{2 k}{\tau_{q}^{2}} \Delta V^{1}+\frac{2}{\tau_{q}^{2}}\left(k \tau_{\theta} \Delta-1\right) V^{2}+\left(\frac{k \tau_{\theta}^{2}}{\tau_{q}^{2}} \Delta-\frac{2}{\tau_{q}}\right) V^{3}-d V^{3} & =F^{3} .
\end{aligned}
$$

Eliminating $V^{2}$ and $V^{3}$, we have to solve

$$
\begin{aligned}
& \underbrace{\left(\frac{2 k}{\tau_{q}^{2}}+\frac{2 k \tau_{\theta} d}{\tau_{q}^{2}}+\frac{k \tau_{\theta}^{2} d^{2}}{\tau_{q}^{2}}\right)}_{=: \gamma_{1}} \Delta V^{1}-\underbrace{\left(\frac{2 d}{\tau_{q}^{2}}+\frac{2 d^{2}}{\tau_{q}}+d^{3}\right)}_{=: \delta_{1}} V^{1}= \\
& F^{3}+\frac{2}{\tau_{q}^{2}} F^{1}+\left(\frac{2}{\tau_{q}}+d-\frac{k \tau_{\theta}^{2}}{\tau_{q}^{2}} \Delta\right)\left(d F^{1}+F^{2}\right)-\frac{2 k \tau_{\theta}}{\tau_{q}^{2}} \Delta F^{1} .
\end{aligned}
$$

Let first $F^{1}, F^{2} \in\left(H^{2} \cap H_{0}^{1}\right)(R)$. Hence we consider for $G^{1} \in L^{2}(R)$ the equation

$$
\gamma_{1} \Delta V^{1}-\delta_{1} V^{1}=G^{1}
$$

where $\gamma_{1}, \delta_{1}$ are positive. The existence of $V^{1} \in\left(H^{2} \cap H_{0}^{1}\right)(R)$ solving (3.11) is well known, then $V^{2}$ and $V^{3}$ both in $\left(H^{2} \cap H_{0}^{1}\right)(R)$ can be determined from the equations (3.7), and (3.8), respectively, and $V \in D(A)$ will solve $(A-d) V=F$. By a density argument using the continuity of $(A-d)^{-1}$ we conclude the solvability for any $F \in \mathcal{H}$.

QED

Now we conclude from the last two Lemmata that $A$ generates a $C_{0}$-semigroup, and hence the initial (boundary) value problem (3.5) is uniquely solvable:

Theorem 3.3 For any $F \in C^{0}([0, \infty), D(A))$ or $F \in C^{1}([0, \infty), \mathcal{H})$ and any $V^{0} \in D(A)$ there is a unique solution $V$ to (3.5) with $V \in C^{1}([0, \infty), \mathcal{H}) \cap C^{0}([0, \infty), D(A))$.

The well-posedness consideration in this section extend naturally to other domains $\Omega \subset$ $\mathbb{R}^{n}, n=1,2,3$, instead of the three-dimensional cylinder $R$. 


\section{Exponential stability}

The aim of this section is to show that the solutions of the problem decay in an exponential way whenever we assume that $\tau_{\theta}>\tau_{q}$. In this Section we assume $g=0$.

From relation (2.6), we know that the following relation

$$
\begin{gathered}
k\left(\hat{\theta}_{, i} \hat{\theta}\right)_{, i}=k|\nabla \hat{\theta}|^{2}+\frac{B \tau_{\theta}^{2}}{2}|\ddot{\theta}|^{2}+\left(C \tau_{\theta}-B\right)|\dot{\theta}|^{2} \\
+\frac{1}{2} \frac{d}{d t}\left(A|\hat{\theta}|^{2}+\tau_{\theta}\left(B+\frac{C \tau_{\theta}}{2}\right)|\dot{\theta}|^{2}+C \theta^{2}+2 B \theta \dot{\theta}\right),
\end{gathered}
$$

is satisfied for a solution.

As we assume that $\tau_{\theta}>\tau_{q}$ we note that

$$
C \tau_{\theta}=\tau_{\theta}-\frac{\tau_{q}^{2}}{\tau_{\theta}}>\tau_{q}-\frac{\tau_{q}^{2}}{\tau_{\theta}}=B
$$

and that the matrix

$$
P:=\left(\begin{array}{cc}
B \tau_{\theta}+\frac{C \tau_{\theta}^{2}}{2} & B \\
B & C
\end{array}\right)
$$

is positively definite. If we define the function

$$
F(t)=\frac{1}{2} \int_{R}\left(A|\hat{\theta}|^{2}+\left(B \tau_{\theta}+\frac{C \tau_{\theta}^{2}}{2}\right)|\dot{\theta}|^{2}+C|\theta|^{2}+2 B \theta \dot{\theta}\right) d V,
$$

we see that

$$
F^{\prime}(t)=-\int_{R}\left(k|\nabla \hat{\theta}|^{2}+\frac{B \tau_{\theta}}{2}|\ddot{\theta}|^{2}+\left(C \tau_{\theta}-B\right)|\dot{\theta}|^{2}\right) d V,
$$

It is clear from the positivity of $P$ that we can obtain two positive constants $M$ and $m$ such that

$$
m \int_{R}\left(|\ddot{\theta}|^{2}+|\dot{\theta}|^{2}+|\theta|^{2}\right) d V \leq F(t) \leq M \int_{R}\left(|\ddot{\theta}|^{2}+|\dot{\theta}|^{2}+|\theta|^{2}\right) d V .
$$

Poincaré's inequality implies that

$$
-\int_{R}|\nabla \hat{\theta}|^{2} d V \leq-C \int_{R}|\hat{\theta}|^{2} d V
$$

where $C$ is a positive constant which depends on the cross-section of $R$ and can be calculated. Thus, observing (4.2) we get the existence of a positive constant $N$ such that

$$
F^{\prime}(t) \leq-N \int_{R}\left(|\ddot{\theta}|^{2}+|\dot{\theta}|^{2}+|\theta|^{2}\right) d V
$$

It follows the existence of a positive constant $K$ such that

$$
F^{\prime}(t)+K F(t) \leq 0
$$


This inequality leads to the exponential decay estimate

$$
F(t) \leq F(0) \exp (-K t)
$$

Thus, observing (4.6), we have proved for

$$
E(t):=\int_{R}\left(|\ddot{\theta}|^{2}+|\dot{\theta}|^{2}+|\theta|^{2}\right) d V
$$

Theorem 4.1 Let $\theta$ be a solution of the problem determined by equation (2.1), the initial conditions (2.3) and the boundary conditions (3.4). Then, the energy term E decays exponentially,

$$
\exists c_{0}, c_{1}>0 \forall t \geq 0: E(t) \leq c_{0} \mathrm{e}^{-c_{1} t} E(0)
$$

\section{Some spatial estimates}

In this Section we establish results on the spatial evolution of solutions of (2.1)-(2.2), (2.4), (2.5) provided that the initial data (2.3) is assumed to be zero. To this end we only need that the two parameters $\tau_{q}$ and $\tau_{\theta}$ are positive. It is worth recalling that the state of art of the spatial behavior of several thermal problems can be found in $[8,9,10,11,12,15,16]$.

The analysis begins by considering the function

$$
F_{\gamma}(z, t)=\int_{0}^{t} \int_{D(z)} \exp (-\gamma s) k \hat{\theta}_{, 3} \tilde{\theta} d A d s
$$

where the positive constant $\gamma$ satisfies (2.12).

We have, using the differential equation (2.1) and partial integration,

$$
\begin{gathered}
F_{\gamma}(z, t)=F_{\gamma}(0, t)+\frac{1}{2} \int_{0}^{z} \int_{D(\eta)} \exp (-\gamma t)\left((\tilde{\theta})^{2}+k\left(\left(\tau_{q}+\tau_{\theta}\right)|\nabla \theta|^{2}+\frac{1}{2}\left(\tau_{q}+\tau_{\theta}\right) \tau_{q} \tau_{\theta}|\nabla \dot{\theta}|^{2}\right)\right) d V \\
+\frac{\gamma}{2} \int_{0}^{t} \int_{0}^{z} \int_{D(\eta)} \exp (-\gamma s)\left((\tilde{\theta})^{2}+k\left(\left(\tau_{q}+\tau_{\theta}\right)|\nabla \theta|^{2}+\frac{1}{2}\left(\tau_{q}+\tau_{\theta}\right) \tau_{q} \tau_{\theta}|\nabla \dot{\theta}|^{2}\right)\right) d V d s \\
+\gamma k \int_{0}^{t} \int_{0}^{z} \int_{D(\eta)} \exp (-\gamma s)\left(\frac{1}{\gamma}|\nabla \theta|^{2}+\frac{\tau_{\theta} \tau_{q}}{\gamma}|\nabla \dot{\theta}|^{2}+\frac{\tau_{q}^{2} \tau_{\theta}^{2}}{4 \gamma}|\nabla \ddot{\theta}|^{2}+\frac{\tau_{q}^{2}+\tau_{\theta}^{2}}{2 \gamma} \nabla \ddot{\theta} \nabla \theta\right) d V d s .
\end{gathered}
$$

Thus,

$$
\begin{gathered}
\frac{\partial F(z, t)}{\partial z}=\frac{\gamma}{2} \int_{0}^{t} \int_{D(z)} \exp (-\gamma s)\left((\tilde{\theta})^{2}+k\left(\left(\tau_{q}+\tau_{\theta}\right)|\nabla \theta|^{2}+\frac{1}{2}\left(\tau_{q}+\tau_{\theta}\right) \tau_{q} \tau_{\theta}|\nabla \dot{\theta}|^{2}\right)\right) d A d s \\
+\gamma k \int_{0}^{t} \int_{D(z)} \exp (-\gamma s)\left(\frac{1}{\gamma}|\nabla \theta|^{2}+\frac{\tau_{\theta} \tau_{q}}{\gamma}|\nabla \dot{\theta}|^{2}+\frac{\tau_{q}^{2} \tau_{\theta}^{2}}{4 \gamma}|\nabla \ddot{\theta}|^{2}+\frac{\tau_{q}^{2}+\tau_{\theta}^{2}}{2 \gamma} \nabla \ddot{\theta} \nabla \theta\right) d A d s .
\end{gathered}
$$




$$
+\frac{1}{2} \int_{D(z)} \exp (-\gamma t)\left((\tilde{\theta})^{2}+k\left(\left(\tau_{q}+\tau_{\theta}\right)|\nabla \theta|^{2}+\frac{1}{2}\left(\tau_{q}+\tau_{\theta}\right) \tau_{q} \tau_{\theta}|\nabla \dot{\theta}|^{2}\right)\right) d A
$$

Our next step is to establish an inequality between $\left|F_{\gamma}(z, t)\right|$ and spatial derivatives of $F_{\gamma}(z, t)$. Applying Schwarz's inequality in (5.1), we get

$$
\begin{gathered}
\left|F_{\gamma}(z, t)\right| \leq \frac{1}{2} \int_{0}^{t} \int_{D(z)}\left[\epsilon^{-1}(\tilde{\theta})^{2}+\epsilon k^{2} \hat{\theta}_{, 3} \hat{\theta}_{, 3}\right] d A d s \\
\leq \frac{1}{2} \int_{0}^{t} \int_{D(z)}\left(\epsilon^{-1}(\tilde{\theta})^{2}+\frac{\epsilon k\left(4+4 \tau_{\theta}^{2}+\tau_{\theta}^{4}\right)}{\lambda_{\gamma}}\left[k \lambda_{\gamma}\left(|\nabla \theta|^{2}+|\nabla \dot{\theta}|^{2}+|\nabla \ddot{\theta}|^{2}\right)\right]\right) d A d s
\end{gathered}
$$

where $\epsilon$ is an arbitrary positive constant, and where $\lambda_{\gamma}$ was defined in Section 2. If we take

it follows that

$$
\epsilon=\sqrt{\frac{2 \lambda_{\gamma}}{k\left(4+4 \tau_{\theta}^{2}+\tau_{\theta}^{4}\right)}}
$$

where

$$
\left|F_{\gamma}\right| \leq \gamma^{-1} \beta_{\gamma} \frac{\partial F_{\gamma}}{\partial z}
$$

$$
\beta_{\gamma}=\epsilon^{-1}
$$

This inequality is well-known in the study of spatial decay estimates. It implies that

$$
F_{\gamma} \leq \gamma^{-1} \beta_{\gamma} \frac{\partial F_{\gamma}}{\partial z}, \text { and }-F_{\gamma} \leq \gamma^{-1} \beta_{\gamma} \frac{\partial F_{\gamma}}{\partial z}
$$

From (5.6), we can obtain an alternative of Phragmen-Lindelöf type which states (see [3]) that the solutions either grow exponentially for $z$ sufficiently large or solutions decay exponentially in the form

$$
\mathcal{E}_{\gamma}(z, t) \leq \mathcal{E}_{\gamma}(0, t) \exp \left(-\gamma \beta_{\gamma}^{-1} z\right)
$$

for all $z \geq 0$, where

$$
\begin{gathered}
\mathcal{E}_{\gamma}(z, t)=\frac{1}{2} \int_{R(z)} \exp (-\gamma t)\left((\tilde{\theta})^{2}+k\left(\left(\tau_{q}+\tau_{\theta}\right)|\nabla \theta|^{2}+\frac{1}{2}\left(\tau_{q}+\tau_{\theta}\right) \tau_{q} \tau_{\theta}|\nabla \dot{\theta}|^{2}\right)\right) d V \\
+\frac{\gamma}{2} \int_{0}^{t} \int_{R(z)} \exp (-\gamma s)\left((\tilde{\theta})^{2}+k\left(\left(\tau_{q}+\tau_{\theta}\right)|\nabla \theta|^{2}+\frac{1}{2}\left(\tau_{q}+\tau_{\theta}\right) \tau_{q} \tau_{\theta}|\nabla \dot{\theta}|^{2}\right)\right) d V d s \\
+\gamma k \int_{0}^{t} \int_{R(z)} \exp (-\gamma s)\left(\frac{1}{\gamma}|\nabla \theta|^{2}+\frac{\tau_{\theta} \tau_{q}}{\gamma}|\nabla \dot{\theta}|^{2}+\frac{\tau_{q}^{2} \tau_{\theta}^{2}}{4 \gamma}|\nabla \ddot{\theta}|^{2}+\frac{\tau_{q}^{2}+\tau_{\theta}^{2}}{2 \gamma} \nabla \ddot{\theta} \nabla \theta\right) d V d s .
\end{gathered}
$$

Thus, we have proved:

Theorem 5.1 Let $\theta$ be a solution of the initial-boundary-value problem (2.1), (2.2),(2.4), (2.5) and null initial conditions. Then either the solutions grow exponentially or the estimate (5.7) is satisfied, where $\mathcal{E}_{\gamma}=-F_{\gamma}$ is defined in (5.8).

We note that this result gives an answer to the question proposed by Hetnarski and Ignaczak ([7], p.474) a principle of Saint-Venant's type in this theory. 


\section{A faster spatial estimate}

It is clear that when $\tau_{q}=\tau_{\theta}$ our equation is very similar to the usual heat equation, cp. (3.6). For the classical heat equation we know that the decay is of the type of a exponential of the square of the distance. This suggests that the spatial decay (5.7) could be improved in general. To point out this fact we concentrate our attention to the case that $\tau_{\theta}>\tau_{q}$. In this Section we assume that $\tau_{\theta}>\tau_{q}$ and we will prove that the spatial decay can be controlled by an exponential of the square of the distance.

Let us to define the function

$$
H(z, t)=\frac{k}{2} \int_{0}^{t} \int_{D(z)}|\hat{\theta}|^{2} d A d s .
$$

We have

$$
\frac{\partial H}{\partial z}=k \int_{0}^{t} \int_{D(z)} \hat{\theta}_{, 3} d A d s
$$

and

$$
\begin{gathered}
\frac{\partial^{2} H}{\partial z^{2}}=\int_{0}^{t} \int_{D(z)} k\left(\hat{\theta}_{, i} \hat{\theta}_{, i}+\frac{B \tau_{\theta}^{2}}{2}|\ddot{\theta}|^{2}+\left(C \tau_{\theta}-B\right)|\dot{\theta}|^{2}\right) d A d s \\
+\frac{1}{2} \int_{D(z)}\left(A|\hat{\theta}|^{2}+\tau_{\theta}\left(B+\frac{C \tau_{\theta}}{2}\right)|\dot{\theta}|^{2}+C \theta^{2}+2 B \theta \dot{\theta}\right) d A .
\end{gathered}
$$

We get

$$
H \frac{\partial^{2} H}{\partial z^{2}}-\frac{1}{2}\left(\frac{\partial H}{\partial z}\right)^{2} \geq 2 \lambda_{1} H^{2}+d H \frac{\partial H}{\partial t}
$$

where $\lambda_{1}$ is the first eigenvalue of the cross-section and $d=k^{-1} A$

If we define the (non-negative) functional

$$
P(z, t)=[H(z, t)]^{1 / 2},
$$

we have that $P(z, t)$ satisfies the second-order differential inequality

$$
\begin{gathered}
P_{z z} \geq k^{*} P+d \dot{P}, \quad z>0, \quad t>0, \\
k^{*}=\lambda_{1} .
\end{gathered}
$$

Now, we show that the function $P(z, t)$ satisfying (6.6) can be bounded above by the solutions to a related initial-boundary value problem for the one-dimensional heat equation. The argument here follows that of Horgan et al [11]. By virtue of its definition, $P(z, t)$ satisfies the initial condition

$$
P(z, 0)=0, \quad z \geq 0
$$

and the boundary condition

$$
P(0, t)=\left(k \int_{0}^{t} \int_{D}\left|\hat{g}\left(x_{\alpha}, t\right)\right|^{2} d A d s\right)^{1 / 2} \equiv g(t) \geq 0, t \geq 0
$$


where

$$
g(0)=0
$$

As the aim of this Section is to obtain an estimate for the spatial decay of the solutions, we start with assuming the following asymptotic behavior for $P(z, t)$ :

$$
P(z, t) \rightarrow 0 \quad(\text { uniformly in } t) \quad \text { as } z \rightarrow \infty \text {. }
$$

Thus the temperature field satisfying (2.1), (2.4)-(2.5) and null initial conditions, is assumed to vanish in a weighted mean-square sense as the axial variable tends to infinity.

Let

$$
v(z, t):=\exp \left(\frac{k^{*} t}{d}\right) P(z, t) .
$$

Then it follows from (6.6) (6.10), (6.9) and (6.11) that $v(z, t) \geq 0$, satisfies

$$
\begin{gathered}
\mathcal{L} v \equiv v_{z z}-d \dot{v} \geq 0, \quad z>0, \quad t>0, \\
v(z, 0)=0, \quad z \geq 0, \\
v(0, t)=\exp \left(\frac{k^{*} t}{d}\right) g(t) \geq 0, \quad t \geq 0, \\
v(z, t) \rightarrow 0 \quad \text { (uniformly in } t) \quad \text { as } z \rightarrow \infty .
\end{gathered}
$$

An upper bound for $v(z, t)$ in terms of the solution of an initial-boundary value problem for the one-dimensional heat equation now follows immediately from the maximum principle. Let $w(z, t)$ be such that

$$
\begin{gathered}
\mathcal{L} w=0, \quad z>0, \quad t>0, \\
w(z, 0)=v(z, 0)=0, \quad z \geq 0, \\
w(0, t)=v(0, t)=\exp \left(\frac{k^{*} t}{d}\right) g(t), \quad t \geq 0, \\
w(z, t) \rightarrow 0(\text { uniformly in } t) \text { as } z \rightarrow \infty .
\end{gathered}
$$

The maximum principle for the heat equation now yields

$$
v \leq w, \quad z \geq 0, \quad t \geq 0
$$

and so, from (6.12), we find that

$$
P(z, t) \leq \exp \left(-\frac{k^{*} t}{d}\right) w(z, t)
$$

The representation for $w(z, t)$ that is useful for our purposes (see [20]) is

$$
w(z, t)=\exp \left(\frac{k^{*} t}{d}\right) g(t) G(z, t)
$$


where the non-negative function $G(z, t)$ is given by

$$
2 G(z, t)=\exp (-\sqrt{k} z) \operatorname{erfc}\left\{\frac{d^{1 / 2} z}{2 t^{1 / 2}}-\left(\frac{k^{*} t}{d}\right)^{1 / 2}\right\}+\exp \left(\sqrt{k^{*}} z\right) \operatorname{erfc}\left\{\frac{d^{1 / 2} z}{2 t^{1 / 2}}+\left(\frac{k^{*} t}{d}\right)^{1 / 2}\right\}
$$

Here the complementary error function $\operatorname{erfc}(x)$ is defined by

$$
\operatorname{erfc}(x)=2(\pi)^{-1 / 2} \int_{x}^{\infty} e^{-s^{2}} d s
$$

Thus, on using (6.22), we get the upper bound

$$
P(z, t) \leq g(t) G(z, t)
$$

On recalling the definition of $P(z, t)$ in (6.5), the result (6.26) can be written directly in terms of the solution $\theta(\mathbf{x}, t)$ to the original problem as

$$
(H(z, t))^{1 / 2} \leq(H(0, t))^{1 / 2} G(z, t), z \geq 0, \quad t \geq 0
$$

where $G(z, t)$ is given in (6.24).

The result (6.27) provides a weighted mean-square estimate for the solution $\hat{\theta}$ subject to the assumption that $\tau_{\theta}>\tau_{q}$.

The arguments in Horgan et al. [11] can be used to show that the estimate (6.27) implies that the spatial decay of end effects in the transient problem is faster than that for the steady-state. To see this, we use the fact that

$$
\lim _{x \rightarrow-\infty} \operatorname{erfc}(x)=2, \quad \lim _{x \rightarrow \infty} \operatorname{erfc}(x)=0,
$$

and so, since $G(z, t)$ is monotonically increasing in $t$, we obtain

$$
G(z, t) \leq \lim _{\tau \rightarrow \infty} G(z, \tau)=\exp \left(-\sqrt{k^{*}} z\right) .
$$

Thus, we have established that the rate of spatial decay is at least as fast as

$$
\exp \left(-\sqrt{k^{*}} z\right)
$$

This decay rate is (optimal) decay rate for the steady-state for $\theta(\mathbf{x})$.

If we employ the inequality

$$
\sqrt{\pi} \operatorname{erfc}(x)<\frac{1}{x} \exp \left(-x^{2}\right), \quad x>0,
$$

(see [1] p.298) in (6.27), then, for $z>2 \sqrt{k^{*}} t / d,(6.24)$ and (6.27) yield the estimate

$$
(H(z, t) d \tau)^{1 / 2} \leq(H(0, t))^{1 / 2} \times\left[\frac{2 d^{3 / 2} z(t / \pi)^{1 / 2} \exp \left(-k^{*} t / d\right)}{d^{2} z^{2}-4 k^{*} t^{2}} \exp \left(-\frac{d z^{2}}{4 t}\right)\right]
$$


This result shows that, for fixed $t$, the spatial decay is ultimately controlled by the factor

$$
\exp \left(-\frac{d z^{2}}{4 t}\right)
$$

rather than the factor $\exp (-\sqrt{k} z)$ found in the steady state case.

A natural question is to relate the estimate for $\hat{\theta}$ with $\theta$. To this end we recall that

$$
\begin{aligned}
\int_{0}^{t}(\hat{f})^{2} d s= & \int_{0}^{t}\left(f^{2}+\frac{\tau_{\theta}^{4}}{4}(\ddot{f})^{2}\right) d s+\left(\tau_{\theta}\left(f^{2}(t)+\tau_{\theta} f(t) \dot{f}(t)+\frac{\tau_{\theta}^{2}}{2} \dot{f}^{2}(t)\right)\right. \\
& -\left(\tau_{\theta}\left(f^{2}(0)+\tau_{\theta} f(0) \dot{f}(0)+\frac{\tau_{\theta}^{2}}{2} \dot{f}^{2}(0)\right) .\right.
\end{aligned}
$$

It is worth noting that the expression

$$
\tau_{\theta}\left(f^{2}(t)+\tau_{\theta} f(t) \dot{f}(t)+\frac{\tau_{\theta}^{2}}{2} \dot{f}^{2}(t)\right)
$$

is positive in the sense that it is equivalent to the measure defined by $f^{2}(t)+\dot{f}^{2}(t)$.

If we assume null initial conditions we obtain the spatial estimate in the usual norm.

\section{Uniqueness in exterior domains}

The aim of this Section is to prove a uniqueness result for the solutions of the equation (2.1) whenever we assume that the solutions do not grow faster than the exponential of the square of the distance from the origin. Thus, we assume here that $R$ is an unbounded domain and that the solutions satisfy the asymptotic conditions

$$
\left|\theta_{, t}, \theta_{, t t}, \theta_{, r}, \theta_{, t r}, \theta_{, t t r}\right| \leq \exp \left(k^{* *} r^{2}\right),
$$

where $k^{* *}$ is a positive constant and $r^{2}=x_{i} x_{i}$.

In this Section we also assume that $\tau_{\theta}>\tau_{q}$. To prove a uniqueness result, it is sufficient to show that the only solution of the null initial and boundary conditions is the null solution.

We define the function

$$
F(t)=\frac{1}{2} \int_{R} \exp \left(-\frac{r^{2}}{\alpha-2 \beta t}\right)\left(A|\hat{\theta}|^{2}+\tau_{\theta}\left(B+\frac{C \tau_{\theta}}{2}\right)|\dot{\theta}|^{2}+C \theta^{2}+2 B \theta \dot{\theta}\right) d V .
$$

where

$$
0<\alpha<\frac{1}{k^{* *}}, \quad \beta:=\frac{k}{A}
$$

In view of the conditions (7.1) on the asymptotic behavior, this function is well defined whenever $t$ is sufficiently small. We have

$$
F^{\prime}(t)=I_{1}+I_{2}
$$


where

$$
I_{1}=-\frac{\beta}{(\alpha-2 \beta t)^{2}} \int_{R} r^{2} \exp \left(-\frac{r^{2}}{\alpha-2 \beta t}\right)\left(A|\hat{\theta}|^{2}+\tau_{\theta}\left(B+\frac{C \tau_{\theta}}{2}\right)|\dot{\theta}|^{2}+C \theta^{2}+2 B \theta \dot{\theta}\right) d V,
$$

and

$$
I_{2}=\frac{1}{2} \int_{R} \exp \left(-\frac{r^{2}}{\alpha-2 \beta t}\right) \frac{d}{d t}\left(A|\hat{\theta}|^{2}+\tau_{\theta}\left(B+\frac{C \tau_{\theta}}{2}\right)|\dot{\theta}|^{2}+C \theta^{2}+2 B \theta \dot{\theta}\right) d V .
$$

We have, using (4.1) and the Dirichlet boundary condition,

$$
I_{2}=J_{1}+J_{2}
$$

where

$$
J_{1}=\frac{2 k}{\alpha-2 \beta t} \int_{R} \exp \left(-\frac{r^{2}}{\alpha-2 \beta t}\right) \hat{\theta} \nabla \hat{\theta} \cdot \mathbf{x} d V
$$

and

$$
J_{2}=-\int_{R} \exp \left(-\frac{r^{2}}{\alpha-2 \beta t}\right)\left(k|\nabla \hat{\theta}|^{2}+\frac{B \tau_{\theta}^{2}}{2}|\ddot{\theta}|^{2}+\left(C \tau_{\theta}-B\right)|\dot{\theta}|^{2}\right) d V .
$$

This implies

$$
J_{1} \leq \frac{k}{A(\alpha-2 \beta t)^{2}} \int_{R} r^{2} \exp \left(-\frac{r^{2}}{\alpha-2 \beta t}\right) A|\hat{\theta}|^{2} d V+\int_{R} \exp \left(-\frac{r^{2}}{\alpha-2 \beta t}\right) k|\nabla \hat{\theta}|^{2} d V .
$$

and by the choice of $\beta$, we conclude

$$
F^{\prime}(t) \leq 0
$$

for every $t$. After integration we obtain

$$
F(t) \leq F(0)
$$

As we assume null initial conditions we obtain that $\hat{\theta}=0$ in a interval with non zero measure. It is clear that the only solution to the problem determined by the equation $\hat{\theta}=0$ with null initial conditions is the null solution and then we obtain that $\theta \equiv 0$ in a interval. We can repeat this argument to obtain that $\theta=0$ for all $t \geq 0$ and the the uniqueness result is proved.

\section{References}

[1] M. Abramowitz I. A. Stegun (Eds.), Handbook of mathematical functions, Dover, New York, 1965. 
[2] DS. Chandrasekharaiah, Hyperbolic thermoelasticity: A review of recent literature, Appl. Mech. Rev., 51(1998), pp. 705-729.

[3] J. N. Flavin, R. J. Knops, L. E. Payne, Decay estimates for the constrained elastic cylinder of variable cross section. Quart. Appl. Math., 47(1989), pp. 325-350.

[4] F. Franchi, B. Straughan, Continuous dependence on the relaxation time and modelling, and unbounded growth in theories of heat conduction with finite propagation speeds, J. Math. Anal. Appl., 185(1994), pp. 726-746.

[5] A.F. Ghaleb, M. Sh. El-Deen Mohamedein, A heat conduction equation with three relaxation times, Int. J. Eng. Sci., 27(1989), pp. 1367-1377.

[6] J. Goldstein, Semigroups of Linear Operators and Applications, Oxford University Press, Oxford, 1985.

[7] R. B. Hetnarski, J. Ignaczak, Generalized thermoelasticity, J. Thermal Stresses, 22(1999), pp. $451-470$.

[8] C. O. Horgan, Recent developments concerning Saint-Venant's principle: An update, Appl. Mech. Rev., 42(1989), pp. 295-303.

[9] C. O. Horgan, Recent developments concerning Saint-Venant's principle: A second update, Appl. Mech. Rev. 49(1996), pp. 101-111.

[10] C. O. Horgan, J. K. Knowles, Recent developments concerning Saint-Venant's principle, J. W. Hutchinson, T. Y. Wu, eds., Adv. Appl. Mech., 23(1983), pp. 179-269.

[11] C. O. Horgan, L. E. Payne, L. T. Wheeler, Spatial decay estimates in transient heat conduction, Quart. Appl. Math., 42(1984), pp. 119-127.

[12] C. O. Horgan, R. Quintanilla, Spatial behaviour of solutions of the dual-phase-lag heat equation, Math. Meth. Appl. Sci., 28(2005), pp. 43-57.

[13] D. Jou, J. Casas-Vazquez, G. Lebon, Extended Irreversible Thermodynamics, SpringerVerlag, Berlin, 1996.

[14] I. Müller, T. Ruggeri, Rational and Extended Thermodynamics, Springer-Verlag, NewYork, 1998.

[15] R. Quintanilla, Spatial bounds and growth estimates for the heat equation with three relaxation times, Math. Meth. Appl. Sci., 20(1997), pp. 1335-1344.

[16] R. Quintanilla, Exponential stability in the dual-phase-lag heat conduction theory, J.NonEquilibrium Thermodynamics, 27(2002), pp. 217-227. 
[17] R. Quintanilla, R. Racke, Qualitative aspects in dual-phase-lag thermoelasticity, SIAM J. Appl. Math., 66(2006), pp. 977-1001.

[18] R. Quintanilla, R. Racke, A note on stability of dual-phase-lag heat conduction, Int. J. Heat Mass Transfer, 49(2006), pp. 1209-1213.

[19] R. Quintanilla, B. Straughan, Explosive instabilities in heat transmission, Proc. Roy. Soc. London, Ser. A. 458(2002), pp. 2833-2838.

[20] I. S. Sokolnikoff, and R. M. Redheffer. (1966). Mathematics of physics and modern engineering. McGraw- Hill, New York, ( Second Edition ).

[21] D. Y. Tzou, A unified approach for heat conduction from macro to micro-scales, J. Heat Transfer, 117(1995), pp. 8-16.

[22] D. Y. Tzou, The generalized lagging response in small-scale and high-rate heating, Int. J. Heat Mass Transfer, 38(1995), pp. 3231-3234. 